Pacific Journal of Mathematics

ON THE LOCATION OF ZEROES OF OSCILLATORY 


\section{ON THE LOCATION OF ZEROES OF OSCILLATORY SOLUTIONS OF $y^{(n)}=c(x) y$}

\section{H. GINGOLD}

We locate the zeroes of oscillatory solutions of wide classes of differential equations, $y^{(n)}=c(x) y$. Asymptotic techniques are used. The asymptotic behaviour of solutions and their derivatives up to the $n$th order are also provided.

New results are obtained in addition to old results becoming more transparent.

1. Introduction. The main purpose of this paper is to demonstrate a method for locating the zeroes of oscillatory solutions of the differential equation

$$
y^{(n)}=c(x) y .
$$

As shown by the references cited, the differential equation (1.1) attracted a considerable amount of attention. However, the location of zeroes of oscillatory solutions of (1.1) does not seem to be available in the literature. It is the purpose of this paper to fill this gap for a wide class of differential equations (1.1).

The method to be used exploits concepts of classical asymptotics which seem to us the most appropriate ones to handle problems of singular differential equations. The singularity of the differential equation (1.1) stems from the fact that the independent variable $x$ ranges on an infinite interval and also from the fact that $c(x)$ may be unbounded.

We do assume an amount of smoothness on the coefficient $c(x)$ which is more restrictive than a continuity assumption made e.g. by Kim [12]. However, this is a reasonable price to be paid for obtaining the fine structure of $y^{(\nu)}(x), \nu=0,1, \ldots, n-1$ as $x \rightarrow \infty$.

In particular, most of the asymptotic properties known so far on oscillatory and nonoscillatory solutions of (1.1) can be better understood by the techniques employed in this work.

An additional reward of this paper is that we produce Prufer type representatives for solutions of (1.1) which belong to certain subspaces of the linear space of solutions of (1.1). See e.g. Hille [9] p. 394.

The course of events in this paper will be as follows: After this introduction we proceed to $\S 2$, which contains preparations for an 
asymptotic decomposition theorem. In $\S 3$ we prove an asymptotic decomposition theorem, and in $\S 4$ we find the location of zeroes of oscillatory solutions of (1.1). This paper is an attempt to generalize results of Gingold [5] for the case $n=2$. Results of $\S 4$, in this paper, depend heavily on $\S 5$ in Gingold [5].

2. Preliminaries. We first convert the scalar differential equation (1.1) into a vectorial differential system for the vector

$$
\left[\begin{array}{c}
y \\
y^{\prime} \\
\vdots \\
y^{(n-1)}
\end{array}\right] \text {. }
$$

The resulting matrix differential system is

$$
Y^{\prime}=A Y
$$

with

$$
A=\left[\begin{array}{lllll}
0 & 1 & & & \\
& & 1 & & \\
& & & \ddots & \\
c(x) & 0 & . & \cdots & 0
\end{array}\right], \quad A=\left(a_{j k}\right), j, k=1, \ldots, n .
$$

In this matrix

$$
\begin{aligned}
& a_{n 1}=c(x), \\
& a_{j, j+1} \equiv 1, \quad j=1, \ldots, n-1
\end{aligned}
$$

and all other entries in this matrix are zero.

Notation 2.1. We will denote

$$
r:=r(x)=|c(x)|^{1 / n} \text {. }
$$

It is a simple exercise to verify that the eigenvalues of the matrix $A$ denoted by $\lambda_{k}(x), k=1, \ldots, n$, are easily found to be

$$
\lambda_{k}(x)=r(x)\left(e^{i \theta_{k}}\right)=r(x)\left(\cos \theta_{k}+i \sin \theta_{k}\right),
$$

where

(2.8) $\boldsymbol{\theta}_{k}=\frac{\arg c(x)}{n}+\frac{2 \pi}{n}(k-1), \quad k=1, \ldots, n, \quad c(x) \neq 0$,

and $\arg z$ is defined for $z$ complex

$$
-\pi<\arg z=\operatorname{Im} \ln z \leq \pi .
$$


We use the following asertion throughout this paper and we will not restate it in the sequel unless it will be replaced.

Assumption 2.2. Let $J$ be the interval $[a, \infty)$. Then

$$
|c(x)| \neq 0, \quad c(x) \text { is real for } x \in J .
$$

(ii) $c(x) \in C^{2}(J)$.

It is a simple consequence of Assumption 2.2 that $r(x) \in C^{2}(J)$. It is also a simple exercise to verify that the matrix $A$ possesses $n$ linearly independent eigenvectors which form the columns of the following Vandermonde matrix $V$,

$$
\begin{aligned}
& V=\left(v_{l k}\right), \quad l, k=1, \ldots, n, \\
& v_{l k}=\lambda_{k}^{l-1}(x) .
\end{aligned}
$$

Therefore, the transformation

$$
Y=V Z
$$

takes the differential equation (2.2) into

$$
Z^{\prime}=A_{1} Z
$$

with

$$
A_{1}=\left[V^{-1} A V-V^{-1} V^{\prime}\right]
$$

and with

$$
V^{-1} A V=\operatorname{diag}\left\{\lambda_{1}(x), \ldots, \lambda_{n}(x)\right\} .
$$

We will need more properties of the matrix $V^{-1} V^{\prime}$ in (2.15). Therefore we will need the following lemma:

Lemma 2.3. Denote by $K$ a constant matrix,

$$
K=\left(k_{s d}\right), \quad s, d=1, \ldots, n,
$$

to be introduced later. Denote by $b$ also a constant to be introduced later. Then if $V$ is the Vandermonde matrix given by (2.11), $V$ is differentiable and

$$
V^{-1} V^{\prime} \equiv(\ln r)^{\prime} b K \text {. }
$$

Proof. In order to find explicitly $V^{-1} V^{\prime}$ we let

(2.19) $\quad V=\left[\begin{array}{llll}1 & 1 & \cdots & 1 \\ \left(e^{i \theta_{1}}\right) r & \left(e^{i \theta_{2}}\right) r & \cdots & \left(e^{i \theta_{r}}\right) r \\ \left(e^{2 \imath \theta_{1}}\right) r^{2} & \left(e^{2 \imath \theta_{2}}\right) r^{2} & \cdots & \left(e^{2 \imath \theta_{n}}\right) r^{2} \\ \vdots & \vdots & & \vdots \\ \left(e^{(n-1) i \theta_{1}}\right) r^{n-1} & \left(e^{(n-1) \theta_{2}}\right) r^{n-1} & \cdots & \left(e^{(n-1) \theta_{n}}\right) r^{n-1}\end{array}\right]$. 
Then

$$
\text { Det } V=r^{[1+2+\cdots+(n-1)]}\left(\operatorname{Det} V_{1}\right)
$$

with,

$$
V_{1}=\left[\begin{array}{llll}
1 & 1 & \cdots & 1 \\
e^{i \theta_{1}} & e^{i \theta_{2}} & & e^{i \theta_{n}} \\
e^{2 i \theta_{1}} & e^{2 i \theta_{2}} & & e^{2 i \theta_{n}} \\
\vdots & \vdots & & \vdots \\
e^{(n-1) i \theta_{1}} & e^{(n-1) i \theta_{2}} & & e^{(n-1) i \theta_{n}}
\end{array}\right]
$$

Thus, one can verify that

$$
\text { Det } V=r^{[n(n-1)] / 2}\left[\prod_{k<l}\left(e^{i \theta_{l}}-e^{i \theta_{k}}\right)\right] \text {. }
$$

Next we turn to the adjugant of $V$ in order to find $V^{-1}$. To this end we evaluate $c_{l k}$ the cofactors of $V$ as follows: Let $V_{l k}$ be the following $(n-1) \times(n-1)$ matrix which is obtained from $V$ by eliminating the $l$ th row and the $k$ th column in an obvious manner. (The arrows point respectively to the eliminated row and column in (2.23)).

$$
\begin{aligned}
& \text { column } k
\end{aligned}
$$

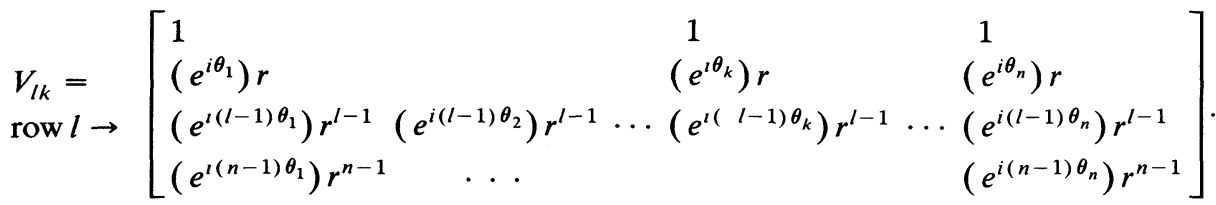

Then,

$$
c_{l k}=(-1)^{l+k} \frac{r^{[1+2+\cdots+(n-1)]}}{r^{(l-1)}}\left(\operatorname{Det} \tilde{V}_{k}\right)
$$

with

$$
\begin{aligned}
& \text { column } k \\
& \tilde{V}_{l k}=\left[\begin{array}{lllll}
1 & & 1 & & 1 \\
e^{i \theta_{1}} & & e^{i \theta_{k}} & & e^{i \theta_{n}} \\
\vdots & & \vdots & & \vdots \\
e^{i(l-1) \theta_{1}} & \cdots & e^{i(l-1) \theta_{k}} & \cdots & e^{i(l-1) \theta_{n}} \\
\vdots & & & & \\
e^{i(n-1) \theta_{1}} & & & \cdots & e^{i(n-1) \theta_{n}}
\end{array}\right]
\end{aligned}
$$


Obviously (Det $\tilde{V}_{l k}$ ) is a constant. Therefore

$$
c_{l k}=(-1)^{l+k} r^{[n(n-1) / 2-(l-1)]}\left(\operatorname{Det} \tilde{V}_{l k}\right) \text {. }
$$

The entries of $V^{-1}$ are given by

$$
(\operatorname{Det} V) V^{-1}=\left(\hat{v}_{s j}\right), \quad s, j=1, \ldots, n,
$$

with

$$
\hat{v}_{s j}=c_{j s} \text {. }
$$

By (2.19) one has

$$
V^{\prime}=\left(v_{l k}^{\prime}\right), \quad v_{l k}^{\prime}=(l-1) e^{i(l-1) \theta_{k}} r^{(l-2)} r^{\prime} .
$$

Denote by $u_{s d}, s, d=1, \ldots, n$ the entries of

$$
(\text { Det } V) V^{-1} V^{\prime}=\left(u_{s d}\right) \text {. }
$$

Then

$$
\text { (2.31) } \begin{aligned}
u_{s d} & =\sum_{j=1}^{n} \hat{v}_{s j} v_{j d}^{\prime}=\sum_{j=1}^{n} c_{j s} v_{j d}^{\prime} \\
& =\sum_{j=1}^{n}(-1)^{j+s} r^{[n(n-1) / 2-(j-1)]}\left(\operatorname{Det} \tilde{v}_{j s}\right)(j-1) e^{i(j-1) \theta_{d}} r^{(j-2)} r^{\prime} \\
& =r^{[n(n-1) / 2]} r^{-1} r^{\prime} \sum_{j=1}^{n}(-1)^{j+s}(j-1) e^{i(J-1) \theta_{d}}\left(\operatorname{Det} \tilde{V}_{j s}\right)
\end{aligned}
$$

We now let

$$
k_{s d}=\sum_{j=1}^{n}(-1)^{j+s}(j-1) e^{i(j-1) \theta_{d}}\left(\operatorname{Det} \tilde{V}_{J s}\right)
$$

and

$$
b^{-1}:=\left[\prod_{k<l}\left(e^{i \theta_{l}}-e^{i \theta_{k}}\right)\right]
$$

and the result follows.

Throughout this work, we will assume that a suitable norm \| $\|$ defined on the set of $n$ by $n$ matrices is given.

We now plan to perform a second linear transformation. To this end, we need the following:

Assumption 2.4. The nonnegative mapping $h(x)$ given by

$$
h(x):=\int_{x}^{\infty}\left|\left(r^{-2} r^{\prime}\right)^{\prime}\right| d t
$$

is finite on $\mathrm{J}$. Also,

$$
\lim _{x \rightarrow \infty}\left(r^{-2}(x) r^{\prime}(x)\right)^{(k)}=0, \quad k=0,1,
$$


and (therefore, without loss of generality) all eigenvalues of $r^{-1} A_{1}$ are distinct on $J$.

We can now provide the following needed lemma:

Lemma 2.5. Consider the matrix $r^{-1} A_{1}$. Let assumptions 2.2, 2.4 hold. Then, there exists on $J$ an $n$ by $n$ matrix function $T(x)$ with the following proeprties;

$T(x)$ is invertible and continuously differentiable on $J$.

$$
T(x)=I+\Delta(x)
$$

where

$$
\|\Delta(x)\|=\mathcal{O}(h(x)), \quad x \rightarrow \infty .
$$

Also,

(2.38) $\tilde{\Omega}:=T^{-1}(x)\left[r^{-1}(x) A_{1}(x)\right] T(x)=\operatorname{diag}\left\{\mu_{1}(x), \ldots, \mu_{n}(x)\right\}$

where $\mu_{J}(x), j=1, \ldots, n$, are the $n$ distinct eigenvalues of $r^{-1} A_{1}$ with the ordering

$$
\lim _{x \rightarrow \infty} \mu_{j}(x)=e^{i \theta_{j}}, \quad j=1, \ldots, n
$$

Moreover,

$$
\left\|T^{\prime}(x)\right\|=\mathcal{O}\left(h^{\prime}(x)\right), \quad x \rightarrow \infty .
$$

Proof. A proof follows from the discussions in Gingold $[6,7]$.

We proceed to the next section.

3. Asymptotic decomposition. In this section, we intend to find a fundamental solution of (2.2) by use of an "asymptotic decomposition theorem."

TheOREM 3.1. Let assumptions 2.2, 2.4 hold. Assume that throughout J $r^{\prime}(x)$ does not change sign if $\ln r(x)$ is unbounded and

$$
\sum_{j \neq \nu}\left|\operatorname{Re} b\left(k_{j j}-k_{\nu \nu}\right)\right| \neq 0, \quad j, k=1, \ldots, n .
$$

Let

$$
\int_{a}^{\infty}\left|\left(r^{-3 / 2} r^{\prime}\right)^{2}\right| d s<\infty
$$

Then, there exists an interval $\hat{J}=[\hat{a}, \infty), a \leq \hat{a}$, such that a fundamental solution of (2.2) on $\hat{J}$ is given by

$$
Y=V(I+P(x)) \exp \int^{x} D(s) d s .
$$


The $n$ by $n$ matrix function $P(x)$ is continuously differentiable on $\hat{J}$ and (3.4)

$$
\lim _{x \rightarrow \infty}\|P(x)\|=0 \text {. }
$$

The matrix $D$ is given by

$$
D=\operatorname{diag}\left[r e^{i \theta_{1}}-b(\ln r)^{\prime} k_{11}, \ldots, r e^{i \theta_{n}}-b(\ln r)^{\prime} k_{n n}\right] .
$$

Proof. First we show that on an interval $\hat{J}$, a fundamental solution of (2.2) can be found such that

$$
Y=V(I+\Delta)(I+\tilde{P}) \exp \int^{x} \tilde{D}(s) d s .
$$

In (3.6), $\tilde{P}=\tilde{P}(x)$ is a certain $n$ by $n$ matrix function such that for $x \in \hat{J}$

$$
\|\tilde{P}(x)\|<1 \text { and } \lim _{x \rightarrow \infty}\|\tilde{P}(x)\|=0 \text {. }
$$

The matrix $\tilde{D}$ is a diagonal matrix defined as follows: Denote by $s_{j k}$, $j, k=1, \ldots, n$, the elements of $T^{-1}(x) T^{\prime}(x)$. Denote

$$
\hat{S}=\operatorname{diag}\left[s_{11}, \ldots, s_{n n}\right] \text {. }
$$

Then

$$
\tilde{D}:=r \tilde{\Omega}-\hat{S}
$$

With the help of Lemma 2.5 we notice that the transformation

$$
Z=T Z_{2}
$$

takes the differential system (2.14) into

$$
Z_{2}^{\prime}=\left(T^{-1} A_{1} T-T^{-1} T^{\prime}\right) Z_{2} .
$$

The differential system (3.11) turns out to be an "almost diagonal system". See Gingold [7]. Thus, there exists an interval $\hat{J}$ such that a fundamental matrix solution of (3.11) is given by

$$
Z_{2}=(I+\tilde{P}(x)) \exp \int^{x} \tilde{D}(s) d s
$$

on $\hat{J}$, where $\tilde{P}(x)$ satisfies (3.7). This implies (3.6).

We now intend to show that

$$
\lim _{x \rightarrow \infty} \int_{x}^{\infty}\|D(s)-\tilde{D}(s)\| d s=0 .
$$

Consider the determinant of $r^{-1} A_{1}-\mu I$.

It is a simple exercise to verify that $p_{n}(\mu)$, the characteristic polynomial of $r^{-1} A_{1}$ has the following form:

$$
p_{n}(\mu)=\prod_{j=1}^{j=n}\left(e^{i \theta_{J}}-b k_{j J} r^{-2} r^{\prime}-\mu\right)+\left(r^{-2} r^{\prime}\right)^{2} p_{n-2}(\mu)
$$


where $p_{n-2}(\mu)$ is a polynomial of order $n-2$ in $\mu$. It possesses continuous coefficients in $x$ on the closed interval $[\hat{a}, \infty]$.

Construct the new polynomial

$$
q_{n}(\mu, \varepsilon)=\prod_{j=1}^{j=n}\left(e^{i \theta_{j}}-b k_{j j} r^{-2} r^{\prime}-\mu\right)+\varepsilon p_{n-2}(\mu)
$$

where $\varepsilon$ is a complex number.

Without loss of generality, we may assume that $q_{n}(\mu, \varepsilon)$ possesses distinct eigenvalues on $\hat{J}$ and $|\varepsilon| \leq \varepsilon_{0}$ where $\varepsilon_{0}$ is a small positive number.

Regard the roots of $q_{n}(\mu, \varepsilon)=0$ as functions of $\varepsilon$ (and the parameter $x)$. Then by considering the initial value problems

$$
\text { (3.16) } \frac{d \mu}{d \varepsilon}=-\frac{p_{n-2}(\mu)}{\partial q_{n} / \partial \mu}, \quad \mu(0)=e^{i \theta_{j}}-b k_{j j} r^{-2} r^{\prime}, j=1, \ldots, n,
$$

we deduce that the $n$ distinct roots of $q_{n}(\mu, \varepsilon)$ are holomorphic functions of $\varepsilon$ for $|\varepsilon| \leq \varepsilon_{0}$.

Moreover,

$$
\mu=e^{i \theta_{\jmath}}-b k_{j j} r^{-2} r^{\prime}+\mathcal{O}(\varepsilon), \quad j=1, \ldots, n,
$$

where $O(\varepsilon)$ is uniform with respect to $x$ on $\hat{J}$.

In (3.15), by letting

$$
\varepsilon=\left(r^{-2} r^{\prime}\right)^{2}
$$

we conclude that

$$
r^{-1} \mu_{j}=e^{i \theta_{j}}-b k_{j j} r^{-1} r^{\prime}+\mathcal{O}\left(\left(r^{-2} r^{\prime}\right)^{2}\right) .
$$

We have

$$
\begin{aligned}
\int_{x}^{\infty}\|D(s)-\tilde{D}(s)\| d s \leq & \int_{x}^{\infty}\|D(s)-r(s) \tilde{\Omega}(s)\| d s \\
& +\int_{x}^{\infty}\|\hat{S}(s)\| d s .
\end{aligned}
$$

This implies (3.13) by combining (3.2) and (2.34). Define now

$$
P(x):=(I+\Delta(x))(I+\tilde{P}(x)) \exp \int_{\infty}^{x}[\tilde{D}(s)-D(s)] d s-I
$$

and the result follows.

REMARKS. It is also possible to use a transformation of the independent variable $x$ given by

$$
\eta=\int^{x} r(s) d s
$$


so that (3.6) would also be derived by Levinson's theorem. See [3], p. 93. However, the setting of this theorem is more general.

For theoretical reasons, it seems immaterial to replace (3.6) by (3.3). However (3.3) points out that the eigenvalues of $A_{1}$ need not be calculated. While the explicit construction of $D(x)$ is a relatively simple task, the numerical calculation of $\tilde{D}(s)$ is more laborious. $\tilde{D}(s)$ cannot be found explicitly.

By following the proof in Gingold [7], finer bounds on $\|P(x)\|$ could be found.

For wide classes of mappings $c(s)$, it can be shown that

$$
\|P(x)\|=\mathcal{O}(h(x)), \quad x \rightarrow \infty .
$$

If we add the assumption that $c(x) \in C^{3}(J)$, then it can also be shown that

$$
\|P(x)\|=\mathcal{O}\left(\frac{h^{\prime}(x)}{r(x)}\right), \quad x \rightarrow \infty .
$$

Theoretically, the bounds in (2.37), (3.23), (3.24) "may not be interesting". However, for numerical purposes, accurate bounds may be crucial.

4. The location of zeroes. Thanks to Theorem 3.1 we are able to locate the zeroes of solutions of (1.1). Moreover, as a bonus other asymptotic properties of solutions will follow.

In the sequel, when we will refer to a solution of (1.1) we will have in mind a non-trivial solution of (1.1). Throughout this section we will assume that conditions of Theorem 3.1 are fulfilled.

Let us prove the following lemma.

Lemma 4.1. For $n=1,2, \ldots$ and $n$ is odd, the matrix $A$ possesses $N=(n-1) / 2$ pairs of complex conjugate eigenvalues. If $n$ is even and $c(x)>0, A(x)$ possesses $N=(n-2) / 2$ pairs of complex conjugate eigenvalues but if $c(x)<0$, it possesses $N=n / 2$ pairs of complex conjugate eigenvalues.

Proof. We let

$$
\beta=\frac{\arg c(x)}{n} .
$$

Then $A$ possesses a real eigenvalue iff $\theta_{k} / \pi$ given by (2.8) is an integer. We will consider several cases. 
Case I. $n$ is odd and $c(x)>0$. It is obvious by (2.8) that

$$
\beta=0, \quad \frac{\theta_{k}}{\pi}=\frac{2(k-1)}{n}, \quad k=1, \ldots, n .
$$

Then for $k=1$ we obtain the only real eigenvalue

$$
\lambda_{1}(x)=r(x) \text {. }
$$

Case II. $n$ is odd and $c(x)>0$. Then,

$$
\beta=\frac{\pi}{n}, \quad \frac{\theta_{k}}{\pi}=\frac{2(k-1)}{n}, \quad k=1, \ldots, n,
$$

which implies that the only real eigenvalue is

$$
\lambda_{k}(x)=-r(x), \quad k=\frac{n+1}{2} .
$$

Case III. $n$ is even and $c(x)>0$.

Then by (2.8) the only real eigenvalues are

$$
\lambda_{1}(x)=r(x), \lambda_{n / 2+1}(x)=-r(x)
$$

Case IV. $n$ is even and $c(x)<0$.

Then by (4.4) it follows that no real eigenvalue is possible at all. Lemma 4.1 helps us to adopt the following notations.

Definition 4.2. We say that the differential equation (1.1) belongs to class I, II, III, or IV if $c(x)$ pertains to one of the four cases described in Lemma 4.1.

Notation 4.3. By relabeling indices we may assume without loss of generality that (from the outset, in §1)

$$
\operatorname{Re} \lambda_{1}>\operatorname{Re} \lambda_{2}>\cdots>\operatorname{Re} \lambda_{N}, \quad \lambda_{J+N}=\bar{\lambda}_{j}, j=1, \ldots, N,
$$

where $N$ is the number of pairs of complex conjugate eigenvalues guaranteed by Lemma 4.1. Moreover, we let

$$
\operatorname{Re} \lambda_{n}=\lambda_{n}=r(x)>\operatorname{Re} \lambda_{1}
$$

if (1.1) belongs to class I.

$$
\operatorname{Re} \lambda_{N}>\operatorname{Re} \lambda_{n}=\lambda_{n}=-r(x)
$$

if (1.1) belongs to class II. 
(III)

(4.10)

$$
\begin{aligned}
r(x) & =\lambda_{n}=\operatorname{Re} \lambda_{n}>\operatorname{Re} \lambda_{1}>\operatorname{Re} \lambda_{N}>\operatorname{Re} \lambda_{n-1} \\
& =-r(x)=\lambda_{n-1}
\end{aligned}
$$

if (1.1) belongs to class III. (The case $N=0$ is not excluded from this notation.)

Notation 4.4. We now define the real functions $A_{\nu}(t), B_{\nu}(t)$ as follows:

$$
\begin{aligned}
A_{\nu}(x)+i B_{\nu}(x):=\int^{x}\left[r(t) e^{i \theta_{\nu}}-r^{-1}(t) r^{\prime}(t) k_{\nu \nu}\right] d t & \\
\qquad \nu & =1, \ldots, n .
\end{aligned}
$$

Thus

$$
\begin{aligned}
& A_{\nu}(x)=\int^{x}\left(r(t) \cos \theta_{\nu}-r^{-1}(t) r^{\prime}(t) a_{1 \nu}\right) d t \\
& B_{\nu}(x)=\int^{x}\left(r(t) \sin \theta_{\nu}-r^{-1}(t) r^{\prime}(t) a_{2 \nu}\right) d t
\end{aligned}
$$

where

$$
\begin{aligned}
& a_{1 \nu}:=\operatorname{Re} k_{\nu \nu}, \\
& a_{2 \nu}:=\operatorname{Im} k_{\nu \nu}, \quad \nu=1, \ldots, n .
\end{aligned}
$$

By the convention just made one has the following:

Case I:

(4.16) $\quad A_{n}=\int_{x_{1}}^{x}\left(r(t)-r^{-1}(t) r^{\prime}(t) a_{1 n}\right) d t, \quad x_{1} \in J$,

$$
B_{n}=-a_{2 n} \int_{x_{1}}^{x} r^{-1}(t) r^{\prime}(t) d t=-a_{2 n} \ln \left[r(x) r^{-1}\left(x_{1}\right)\right]
$$

$$
A_{j}=\int_{x_{1}}^{x}\left(r(t) \cos \frac{2 j}{n} \pi-r^{-1}(t) r^{\prime}(t) a_{1 j}\right) d t
$$

$$
B_{j}=\int_{x_{1}}^{x}\left(r(t) \sin \frac{2 j}{n} \pi-r^{-1}(t) r^{\prime}(t) a_{2 j}\right) d t, \quad j=1, \ldots, N .
$$

Case II:

$$
\begin{aligned}
& \text { (4.20) } \quad A_{j}:=\int_{x_{1}}^{x}\left(r(t) \cos \frac{2 j-1}{n} \pi-r^{-1}(t) r^{\prime}(t) a_{1 j}\right) d t, \\
& \text { (4.21) } \quad B_{j}:=\int_{x_{1}}^{x}\left(r(t) \sin \frac{2 j-1}{n} \pi-r^{-1}(t) r^{\prime}(t) a_{2 j}\right) d t, \\
& j=1, \ldots, N .
\end{aligned}
$$




$$
\begin{aligned}
A_{n} & =-\int_{x_{1}}^{x}\left(r(t)+r^{-1}(t) r^{\prime}(t) a_{1 n}\right) d t \\
B_{n} & =-a_{2 n} \ln \left[r(x) r^{-1}\left(x_{1}\right)\right] .
\end{aligned}
$$

Case III. $A_{n}, B_{n}$, are given by (4.16), (4.17). $A_{j}, B_{j}$ are given by (4.18), (4.19). $A_{n-1}, B_{n-1}$ are obtained from (4.22), (4.23) by substituting $n-1$ in place of $n$.

Case IV. $A_{j}, B_{j}$ are given by (4.20), (4.21).

For the sake of uniformity we add the following assumption:

Assumption 4.5. If

$$
\sum_{\nu=1}^{\nu=n}\left|a_{2 \nu}\right|^{2} \neq 0
$$

then the function $r(x)$ is monotone increasing or monotone decreasing on $\hat{J}$.

Since we have Assumption 4.5, we may add the following assumption without loss of generality.

Assumption 4.6. For $t \in \hat{J}$ in cases I, III

$$
\sin \frac{2 \pi}{n}-r^{-n}(t) r^{\prime}(t) a_{1 \nu} \geq 0, \quad \nu=1, \ldots, n,
$$

and in cases I, IV

$$
\sin \frac{\pi}{n}-r^{-2}(t) r^{\prime}(t) a_{1 \nu} \geq 0, \quad \nu=1, \ldots, n .
$$

This assumption is given in order to guarantee that the functions $B_{\nu}(x)$, found above, possess inverses.

Indeed, the following lemma (whose trivial proof is omitted) can be proven.

LeMMA 4.7. With Assumptions 4.5 and 4.6 the functions $B_{n}(x), B_{n-1}(x)$ and $B_{j}(x), j=1, \ldots, N$, are monotone functions of $x$ on $\hat{J}$.

We will need to find $n$ real linearly independent solutions of (1.1). We first define

$$
W=\left(w_{j k}\right)=V(I+P), \quad P=\left(p_{J k}\right), j, k=1, \ldots, n,
$$

and we write down the elements of the first row of $W$ by

$$
w_{1 \nu}=1+\sum_{\nu}, \quad \nu=1,2, \ldots,
$$


with

$$
\sum_{\nu}:=\sum_{j=1}^{n} p_{j \nu}
$$

Similarly, we have

$$
w_{2 \nu}=\sum_{j=1}^{n} \lambda_{J}\left(\delta_{j \nu}+p_{j \nu}\right)=\lambda_{\nu}\left(1+p_{\nu \nu}\right)+\sum_{j \neq \nu}^{n} \lambda_{J} p_{J \nu}
$$

and

$$
\begin{aligned}
w_{m \nu} & =\sum_{j=1}^{n} \lambda_{j}^{m-1}\left(\delta_{j \nu}+p_{j \nu}\right) \\
& =\lambda_{\nu}^{m-1}\left(1+p_{\nu \nu}\right)+\sum_{j \neq \nu}^{n} \lambda_{j}^{m-1} p_{j \nu}, \quad m=2, \ldots, n .
\end{aligned}
$$

We choose $n$ real linearly independent solutions of (1.1) as follows: By (4.28) via (3.3) we have each of the $n$ linearly independent solutions of (1.1) to be

$$
\left|1+\sum_{\nu}\right| \exp \left(A_{\nu}(x)+i \theta_{1 \nu}(x)+i B_{\nu}(x)\right)
$$

with

$$
\theta_{1 \nu}=\arg \left(1+\sum_{\nu}\right), \quad \nu=1, \ldots, n .
$$

From now on the letter $y$ with lower indices will denote something other than the entries of the matrix function $Y$ which appears in (3.3).

Thus, we adopt

Notation 4.8. We denote $n$ real solutions of (1.1) by $y_{\nu}(x), \nu=1 \cdots n$ and we define them as follows:

If (1.1) belongs to classes I, II or III

$$
\begin{aligned}
y_{n}(x) & =\operatorname{Re} w_{1 n} \exp \left(A_{n}+i B_{n}\right) \\
& =\left|1+\sum_{n}\right|\left(\exp A_{n}(x)\right) \cos \left(B_{n}(x)+\theta_{1 n}(x)\right) \\
y_{1 j}(x) & =\operatorname{Re} w_{1 j} \exp \left(A_{j}+i B_{j}\right) \\
& =\left|1+\sum_{j}\right|\left(\exp A_{j}(x)\right) \cos \left(B_{j}(x)+\theta_{i j}(x)\right) \\
y_{2 j}(x) & =\operatorname{Im} w_{1 j} \exp \left(A_{j}+i B_{j}\right) \\
& =\left|1+\sum_{j}\right|\left(\exp A_{j}(x)\right) \sin \left(B_{j}(x)+\theta_{i j}(x)\right), \quad j=1, \ldots, N .
\end{aligned}
$$


If (1.1) belongs to class III, we pick

$$
\text { (4.32) } \begin{aligned}
y_{n-1}(x) & =\operatorname{Re} w_{1, n-1} \exp \left(A_{n-1}+i B_{n-1}\right) \\
& =\left|1+\sum_{n-1}\right|\left(\exp A_{n-1}(x)\right) \cos \left(B_{n-1}(x)+\theta_{1, n-1}(x)\right)
\end{aligned}
$$

and the rest $n-1$ real solutions according to (4.29), (4.30), (4.31). If (1.1) belongs to class IV we pick the $2 N=n$ real solutions according to (4.30), (4.31).

It is simple to verify that in all cases I-IV the set of $n$ real solutions form a basis for the linear space of solutions of (1.1).

Notation 4.9. We denote by $S_{n}$ the linear subspace generated by $y_{n}(x)$ if (1.1) belongs to class I, II, or III. If (1.1) belongs to class IV, $S_{n}$ will denote an empty set.

We denote by $S_{n-1}$ the linear subspace generated by $y_{n-1}(x)$ if (1.1) belongs to class III. If (1.1) belongs to class I, II or IV, $S_{n-1}$ will denote an empty set.

We denote by $S_{j}, j=1, \ldots, N$, the $N$ two-dimensional linear spaces spanned by $y_{1 j}(x), y_{2 j}(x)$. If $N=0$, we let $S_{j}$ above denote empty sets.

We also denote by $S$ the linear space of solutions of (1.1).

Let us first turn to the location of zeroes of a non-trivial solution $y_{j}(x) \in S_{j}, j=1, \ldots, N(N>0)$.

Let $c_{1 j}, c_{2 j}$ be two real variables. Then, it can be easily verified that

$$
\begin{gathered}
y_{j}(x)=c_{1 j}(x)+c_{2 j} y_{2 j}(x) \\
=\left[M_{j}(x) \cos \left(B_{j}(x)+\theta_{1 j}(x)+\Psi_{1,2, j}\right)\right] \sqrt{c_{1 j}^{2}+c_{2 j}^{2}} \\
M_{j}(x):=\left|1+\sum_{j}\right|\left(\exp A_{J}(x)\right)
\end{gathered}
$$

and with

$$
\tan \Psi_{1,2, j}=\frac{-c_{2 J}}{c_{1 j}}, \quad j=1, \ldots, N .
$$

In order to better understand the process of locating the zeroes of $y(x)$, we define an "ideal solution" of (1.1), $y_{L}(x)$ by letting in (4.33), (4.34).

$$
P=0 \text {. }
$$

For the particular solution (4.33) we then get

$$
\boldsymbol{\theta}_{1 j}(x) \equiv 0, \quad \sum_{j} \equiv 0
$$


to obtain

$$
\text { (4.38) } \quad y_{L}(x)=\left(\exp A_{j}(x)\right) \sqrt{c_{1 j}^{2}+c_{2 j}^{2}} \cos \left(B_{j}(x)+\Psi_{1,2, j}\right) \text {. }
$$

We do not claim that $y_{L}(x)$ is a solution of (1.1). However, the location of its zeroes on $\hat{J}$ are easily observed to be at $x_{l}$ such that

$$
B_{j}\left(x_{l}\right)+\Psi_{1,2, j}=(2 l+1) \frac{\pi}{2} \quad(l \text { an integer }),
$$

or if $B_{j}(x)$ has an inverse on $\hat{J}$

$$
x_{l}=B_{J}^{-1}\left((2 l+1) \frac{\pi}{2}-\Psi_{1,2, j}\right) .
$$

We expect the zeroes of $y(x)$ for $x$ large enough, to be close to $x_{1}$ given by (4.39) or by (4.40).

Indeed, we are now able to apply the results of Gingold [5], §5, to locate with high accuracy the zeroes of $y_{j}(x), j=1, \ldots, N$.

It will be superfluous to repeat all the arguments of Gingold [5]. Therefore, we state our results in a form such that the reader who is interested in the details will be able to easily reproduce them as an exercise.

The next theorems provide information on solutions of (1.1) beyond that given by Kim [12, 13], Willet [24], Kreith [15], Swanson [21]. We did not come across any other method in the past which also provided with high accuracy the location of zeroes of solutions of (1.1). It clearly demonstrates an edge of asymptotic techniques.

THEOREM 4.10. Let assumptions of Theorem 3.1 and Assumptions 4.5 and 4.6 hold. Then, the linear subspace $S_{n},\left(S_{n-1}\right)$ contains nonoscillatory solutions of (1.1) if either

$$
a_{2 n}=0 \quad\left(a_{2, n-1}=0\right) .
$$

or (without restriction on $\left.a_{2 n}\left(a_{2, n-1}\right)\right)$ if

$$
\sup _{x \in J}|\ln r(x)|<\infty \text {. }
$$

moreover, $y_{n} \in S_{n}$ has an exponential growth in cases I and III and it has an exponential decay in case II. $y_{n-1} \in S_{n-1}$ has exponential decay in case III. If (1.1) belongs to class III, then

$$
\lim _{x \rightarrow \infty} \frac{M_{n-1}}{M_{n}}=0
$$

where

$$
M_{n}:=M_{n}(x)=\left|1+\sum_{n}\right| \exp A_{n}(x),
$$




$$
M_{n-1}:=M_{n-1}(x)=\left|1+\sum_{n-1}\right| \exp A_{n-1}(x) .
$$

Proof. This is an immediate consequence of the definitions.

We have

THEOREM 4.11. Let assumptions of Theorem 4.10 hold. If $S_{n}$ is not the empty set and $N>0$ then in cases I, III,

$$
\lim _{x \rightarrow \infty} \frac{y_{j}(x)}{M_{n}(x)}=0, \quad j=1, \ldots, N .
$$

If $N>1$,

$$
\lim _{x \rightarrow \infty} \frac{M_{j}(x)}{M_{j+1}(x)}=0, \quad j=1, \ldots, N-1,
$$

where $M_{j}$ are given by (4.34).

If $S_{n-1}$ is not the empty set and $N>0$ then in case III

$$
\lim _{x \rightarrow \infty} \frac{M_{n-1}(x)}{M_{n}(x)}=0 .
$$

Proof. The trivial proof is omitted.

Next we have

THEOREM 4.12. If assumptions of Theorem 4.10 hold. Let $n \geq 3$, or let (1.1) belong to the class IV.

Then $S$ contains $N$ linear subspaces $S_{j}$ of oscillatory solutions $y_{j}$ given by (4.33), $j=1, \ldots, N$.

The location of the zeroes of $y_{j}$ are given at the points $x_{m_{j}}$ on $\hat{J}$ such that

$$
\lim _{|m,| \rightarrow \infty}\left[x_{m j}-B_{j}^{-1}\left(\left(2 m_{j}+1\right) \frac{\pi}{2}-\Psi_{1,2, j}\right)\right]=0
$$

and also

$$
x_{m_{j}} \sim B_{j}^{-1}\left(\left(2 m_{j}+1\right) \frac{\pi}{2}-\Psi_{1,2, j}\right), \quad\left|m_{j}\right| \rightarrow \infty .
$$

$\left|m_{j}\right| \rightarrow \infty$ means that $m_{j}$ attains successive integral values and either $m_{j} \rightarrow+\infty$ or $m_{j} \rightarrow-\infty$.

Moreover, let $a_{2 n} \neq 0$ and let

$$
\lim _{x \rightarrow \infty}|\ln r(x)|=\infty .
$$

Then in cases I, II, $y_{n} \in S_{n}$ is an oscillatory solution with zeroes $x_{m_{n}}$

(4.52) $\lim _{\left|m_{n}\right| \rightarrow \infty}\left[x_{m_{n}}-B_{n}^{-1}\left(\left(2 m_{n}+1\right) \frac{\pi}{2}\right)\right]=0$,

$$
x_{m_{n}} \sim B_{n}^{-1}\left(\left(2 m_{n}+1\right) \frac{\pi}{2}\right),\left|m_{n}\right| \rightarrow \infty .
$$


In cases II, III, with (4.51) and $a_{2, n-1} \neq 0, y_{n-1} \in S_{n-1}$ is an oscillatory solution with zeroes $x_{m_{n-1}}$ satisfying (4.52) with $n-1$ replacing $n$.

\section{Proof. See Gingold [5] §5.}

We state a theorem which summarizes the preceding oscillation results.

THEOREM 4.13. Let assumptions of Theorem 4.10 hold. Let $y(x)$ be the general solution of (1.1) given by

(4.53) $y(x)=c_{n} y_{n}(x)+\left(\sum_{j=1}^{N} c_{1 j} y_{1 j}(x)+c_{2 J} y_{2 J}(x)\right)+c_{n-1} y_{n-1}(x)$.

If $S_{n}$ or $S_{n-1}$ or both are zero we choose correspondingly $c_{n}$ or $c_{n-1}$ or both to be zero.

(i) If

$$
a_{2 n} \neq 0, \quad a_{2, n-1} \neq 0
$$

and (4.51) holds then each non-trivial solution of (1.1) is oscillatory. In particular, all solutions in case IV are oscillatory.

(ii) Let $c_{n} \neq 0$. Then, (in cases I, III) the zeroes of $x_{m_{1}}$ of $y(x)$ are such that with $j=n$ they satisfy (4.52) and

$$
\left|\frac{y(x)-c_{n} y_{n}(x)}{M_{1}(x)}\right| \leq C, \quad x \in \hat{J},
$$

for a certain constant $C$.

(iii) Let

$$
c_{n}=c_{1 j}=c_{2 j}=0, j=1, \ldots, j_{0}-1, j_{0} \leq N .
$$

Then the zeroes of $y(x)$ are located at points $x_{m_{J_{0}}}$ such that

$$
\lim _{m_{J_{0}} \rightarrow \infty}\left[x_{m_{J_{0}}}-B_{J_{0}}^{-1}\left(\left(2 m_{J_{0}}+1\right) \frac{\pi}{2}-\Psi_{1,2, J_{0}}\right)\right]=0
$$

and

$$
x_{m_{j_{0}}} \sim B_{j_{0}}^{-1}\left(\left(2 m_{j_{0}}+1\right) \frac{\pi}{2}-\Psi_{1,2, j_{0}}\right), \quad\left|m_{j_{0}}\right| \rightarrow \infty .
$$

Also

$$
\left|\frac{y(x)-y_{j_{0}}(x)}{M_{l}(x)}\right| \leq C, \quad x \in \hat{J}
$$


with $l=n-1$ if $j_{0}=N$ and $l=j_{0}+1$ if $l<N$, and $C$ a certain constant. (If $S_{n-1}$ is empty put $M_{n-1} \equiv 1$.)

(iv) If in case III

$$
c_{n}=c_{1,}=c_{2,}=0, \quad j=1, \ldots, N,
$$

then the zeroes of $y(x)$ are located at $x_{m_{n-1}}$. This satisfies (4.52) with $n-1$ replacing $n$.

Proof. The inequalities (4.55), (4.59) follow directly from the definitions of $A_{\nu}(x), M_{\nu}(x)$. Thus, in (4.55) we take

$$
C:=\operatorname{Sup}_{x \in \hat{J}}\left|\frac{y(x)-c_{n} y_{n}(x)}{M_{1}(x)}\right|<\infty
$$

and in (4.59) we take

$$
C:=\operatorname{Sup}_{x \in \hat{J}}\left|\frac{y(x)-y_{J_{0}}(x)}{M_{l}(x)}\right|<\infty .
$$

For the approximation of the zeroes of $y(x)$, we "identify in (4.53) a leading term" and use the details in Gingold [5] §5.

Next we obtain a "non-oscillation" theorem.

THEOREM 4.14. Let assumptions of Theorem 4.10 hold.

(i) Let $a_{2 n}=0$ or let $a_{2 n} \neq 0$ and let (4.42) hold.

If $c_{n} \neq 0$ when $S_{n}$ is a non-empty set, then in cases I, III $y(x)$ is non-oscillatory and (4.61) holds.

(ii) If $a_{2, n-1}=0$ or if $a_{2, n-1}=0$ and (4.42) holds and

$$
c_{n}=c_{1,}=c_{2,}=0, \quad j=1, \ldots, N,
$$

then in case III $y(x)$ is nonoscillatory if $S_{n-1}$ is not an empty set.

If in case III, $a_{2 n}=0$ or $a_{2 n} \neq 0$ and (4.42) hold, and if

$$
c_{\imath j}=c_{2 \jmath}=0, \quad j=1, \ldots, N \text {. }
$$

Then $y(x)$ is nonoscillatory.

Proof. We omit the trivial proof.

REMARKS. A similar analysis carries over to $n$th order linear homogeneous differential equations whose characteristic polynomials possesses $n$ distinct eigenvalues. In this case the location of zeroes of oscillatory solutions can be provided in terms of the elements on the main diagonal of a certain matrix derived from the coefficients of the linear differential equation. 


$$
\lim _{x \rightarrow \infty} r^{-2} r^{\prime} \neq 0, \quad \infty
$$

then a different analysis is needed. It is an easy exercise to verify that for

$$
c(x)=\theta x^{m}, \quad m>-n, \theta \text { real, }
$$

all conditions of Theorem 3.1 are satisfied.

The same is true for (4.66) and (4.67).

$$
c(x)=\theta e^{x^{\alpha}}, \quad \theta \text { real and } \alpha>0 .
$$

It is worth noticing that in case (4.66) or in case

$$
c(x)=x^{\alpha}(\ln x)^{\beta}, \quad \alpha>-n, \beta \text { real, }
$$

the "analytic theory" of asymptotic expansions cannot be applied.

This article also shows how to derive Pruffer type formulas for solutions of higher order linear differential equations. This question has been asked by several authors. See Swanson [21], pg. 95.

The relation between oscillation and certain eigenvalue problems of (1.1) is well known. Thus, our method provides approximations to eigenvalues in certain eigenvalue problems.

\section{REFERENCES}

[1] G. V. Anan'eva and V. Balaganski, Oscillation of the solutions of certain differential equations of higher order, Uspehi Mat. Nauk, 14 (1959), 135-140.

[2] G. A. Bogar, Oscillations of nth order differential equations with retarded argument, SIAM J. Math. Anal., 5 (1974), 473-481.

[3] E. A. Coddington and N. Levinson, Theory of Ordinary Differential Equations, McGraw-Hill, N. Y., 1955.

[4] G. J. Etgen and W. E. Taylor, Jr., The essential uniqueness of bounded nonoscillatory solutions of certain even order differential equations, Pacific J. Math., 68 (1977), 339-346.

[5] H. Gingold, On the location of zeroes of oscillatory solutions, Trans. Amer. Math. Soc., 279 (1983), 471-495.

[6] On the global existence of a simplifying transforming matrix, SIAM J. Math. Anal., 9(1978), 1076-1082.

[7] Almost diagonal systems in asymptotic integration, Proc. Edinburgh Math. Soc., (to appear).

[8] P. Hartman, Ordinary Differential Equations, Wiley, New York, 1964.

[9] E. Hille, Lectures on Differential Equations, Addison-Wesley Publishing Co., Reading Mass., 1969.

[10] I. T. Kiguradze, Oscillation properties of solutions of certain ordinary differential equations, Dokl. Akad. Nauk, 144 (1962), 33-36.

[11] On the oscillatory character of solutions of the equation $d^{m} u \mid d t^{m}+$ $a(t)|u|^{n} \operatorname{sign} u=0$, Mat. Sb., 65 (107) (1964), 172-187. 
[12] W. J. Kim, Nonoscillatory solutions of a class of nth-order linear differential equations, J. Differential Equations, 27 (1978), 19-27.

[13] Asymptotic properties of nonoscillatory solutions of higher order differential equations. Pacific J. Math., 93 (1981), 107-114.

[14] V. A. Kondrat'ev, Oscillatory properties of solutions of $y^{(n)}+p y=0$, Trudy Moskov. Mat. Obsc., 10 (1961), 419-436.

[15] K. Kreith, Oscillation Theory, Lecture Notes in Mathematics \# 324, Springer Verlag, Berlin-Heidelberg-New York, 1973.

[16] J. G. Mikusinski, On Fite's oscillation theorem, Colloq. Math., 2 (1949), 34-39.

[17] Z. Nehari, Disconjugacy criteria for linear differential equations, J. Differential Equations, 4 (1968), 604-611.

[18] Nonlinear techniques for linear oscillation problems, Trans. Amer. Math. Soc., 210 (1975), 387-406.

[19] __ Green's functions and disconjugacy, Arch. Rational Mech. Anal., 62 (1976), 53-76.

[20] G. H. Ryder and D. V. Wend, Oscillation of solutions of certain differential equations of nth order, Proc. Amer. Math. Soc., 25 (1970), 463-469.

[21] C. A. Swanson, Comparison and Oscillation Theory of Linear Differential Equations, Academic Press, New York, (1965).

[22] I. M. Sobol', On the asymptotic behaviour of the solutions of linear differential equations, Dokl. Akad. Nauk SSSR, 61 (1948), 219-222.

[23] W. F. Trench, A sufficient condition for eventual disconjugacy, Proc. Amer. Math. Soc., 52 (1975), 139-146.

[24] D. Willett, Disconjugacy tests for singular linear differential equations, SIAM J. Math. Anal., 2 (1971), 536-545.

Received March 3, 1983.

WEST VIRGINIA UNIVERSITY

MORGANTOWN, WV 26506 


\section{PACIFIC JOURNAL OF MATHEMATICS EDITORS}

\author{
V. S. VARADARAJAN (Managing Editor) \\ University of California \\ Los Angeles, CA 90024 \\ Charles R. DePrima \\ California Institute of Technology \\ Pasadena, CA 91125 \\ R. FInN \\ Stanford University \\ Stanford, CA 94305
}

\section{HeRmanN FlaschKa \\ University of Arizona \\ Tucson, AZ 85721}

Ramesh A. Gangolli

University of Washington

Seattle, WA 98195

ROBION KIRBY

University of California

Berkeley, CA 94720

\section{C. MOORE}

University of California

Berkeley, CA 94720

\section{H. SAMELSON}

Stanford University

Stanford, CA 94305

HAROLD STARK

University of California, San Diego

La Jolla, CA 92093

\section{ASSOCIATE EDITORS}
R. ARENS
E. F. BECKENBACH
B. H. NeumanN
F. Wolf
K. Yoshida (1906-1982)

\section{SUPPORTING INSTITUTIONS}
CALIFORNIA INSTITUTE OF TECHNOLOGY

\author{
UNIVERSITY OF SOUTHERN CALIFORNIA \\ STANFORD UNIVERSITY \\ UNIVERSITY OF HAWAII \\ UNIVERSITY OF TOKYO \\ UNIVERSITY OF UTAH \\ WASHINGTON STATE UNIVERSITY \\ UNIVERSITY OF WASHINGTON
}
UNIVERSITY OF OREGON

\begin{abstract}
UNIVERSITY OF ARIZONA
UNIVERSITY OF BRITISH COLUMBIA

UNIVERSITY OF CALIFORNIA

MONTANA STATE UNIVERSITY

UNIVERSITY OF NEVADA, RENO

NEW MEXICO STATE UNIVERSITY

OREGON STATE UNIVERSITY
\end{abstract}

The Supporting Institutions listed above contribute to the cost of publication of this Journal, but they are not owners or publishers and have no responsibility for its content or policies.

Mathematical papers intended for publication in the Pacific Journal of Mathematics should be in typed form or offset-reproduced (not dittoed), double spaced with large margins. Please do not use built up fractions in the text of the manuscript. However, you may use them in the displayed equations. Underline Greek letters in red, German in green, and script in blue. The first paragraph must be capable of being used separately as a synopsis of the entire paper. In particular it should contain no bibliographic references. Please propose a heading for the odd numbered pages of less than 35 characters. Manuscripts, in triplicate, may be sent to any one of the editors. Please classify according to the scheme of Math. Reviews, Index to Vol. 39. Supply name and address of author to whom proofs should be sent. All other communications should be addressed to the managing editor, or Elaine Barth, University of California, Los Angeles, California 90024.

There are page-charges associated with articles appearing in the Pacific Journal of Mathematics. These charges are expected to be paid by the author's University, Government Agency or Company. If the author or authors do not have access to such Institutional support these charges are waived. Single authors will receive 50 free reprints; joint authors will receive a total of 100 free reprints. Additional copies may be obtained at cost in multiples of 50 .

The Pacific Journal of Mathematics is issued monthly as of January 1966. Regular subscription rate: $\$ 190.00$ a year (5 Vols., 10 issues). Special rate: $\$ 66.00$ a year to individual members of supporting institutions.

Subscriptions, orders for numbers issued in the last three calendar years, and changes of address should be sent to Pacific Journal of Mathematics, P.O. Box 969, Carmel Valley, CA 93924, U.S.A. Old back numbers obtainable from Kraus Periodicals Co., Route 100, Millwood, NY 10546.

The Pacific Journal of Mathematics at P.O. Box 969, Carmel Valley, CA 93924 (ISSN 0030-8730) publishes 5 volumes per year. Application to mail at Second-class postage rates is pending at Carmel Valley, California, and additional mailing offices. Postmaster: Send address changes to Pacific Journal of Mathematics, P.O. Box 969, Carmel Valley, CA 93924.

PUBLISHED BY PACIFIC JOURNAL OF MATHEMATICS, A NON-PROFIT CORPORATION

Copyright $\odot 1985$ by Pacific Journal of Mathematics 


\section{Pacific Journal of Mathematics}

Vol. 119, No. $2 \quad$ June, 1985

Mustafa Agah Akcoglu and Meira Falkowitz (Soshniak), A general local

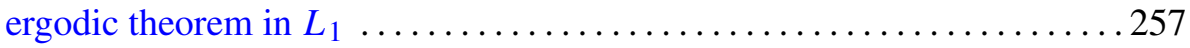

W. Wistar (William) Comfort and Lewis Chandlee Robertson,

Cardinality constraints for pseudocompact and for totally dense

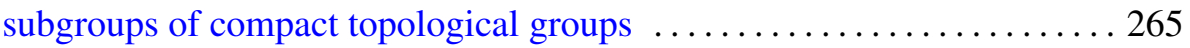

John Morse Delaurentis and Boris G. Pittel, Random permutations and

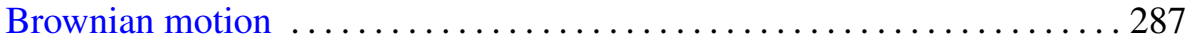

José Esteban Galé, Gel'fand theory in algebras of differentiable functions on Banach spaces ......................................... 303

Harry Gingold, On the location of zeroes of oscillatory solutions of

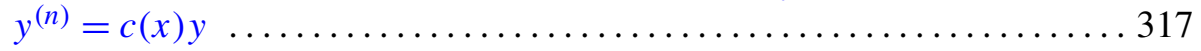

Kei Ji Izuchi, Zero sets of interpolating Blaschke products .............337

Mahesh Nerurkar, Ergodic continuous skew product actions of amenable

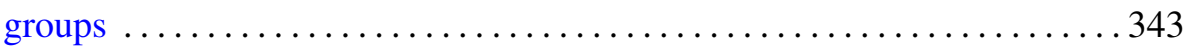

R. Owens, A maximal function characterization of a class of Hardy

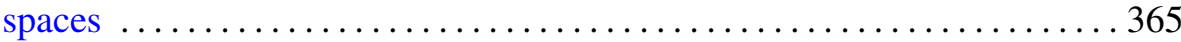

Judith Anne Packer, Point spectrum of ergodic abelian group actions and the corresponding group-measure factors $\ldots \ldots \ldots \ldots \ldots \ldots \ldots \ldots \ldots \ldots \ldots \ldots$

Judith Anne Packer, On the embedding of subalgebras corresponding to quotient actions in group-measure factors $\ldots \ldots \ldots \ldots \ldots \ldots \ldots \ldots . \ldots 4$

Iain Raeburn and Joseph L. Taylor, The bigger Brauer group and étale cohomology

David Rosen, The Diophantine equation $a x+b y=c$ in $Q(\sqrt{5})$ and other number fields

Mau-Hsiang Shih and Kok Keong Tan, Noncompact sets with convex sections

Lee Barlow Whitt, Codimension two isometric immersions between Euclidean spaces

Rodney Ian Yager, Iwasawa theory for the anticyclotomic extension 